\title{
eC-CLEM: Flexible Multidimensional Registration Software for Correlative Microscopies with Refined Accuracy Mapping
}

\author{
Xavier Heiligenstein ${ }^{1,2,5}$, Perrine Paul-Gilloteaux ${ }^{1,2,3,5}$, Martin Belle ${ }^{4}$, Graça Raposo ${ }^{1,2}$, Jean Salamero ${ }^{1,2}$ \\ ${ }^{1 .}$ Cell and Tissue Imaging Facility CNRS Institut Curie, PSL Research University, F-75248 Paris, \\ France \\ 2. CNRS UMR 144, Structure and Membrane Compartments, Paris, France \\ 3. SFR Santé F Bonamy Nantes CNRS INSERM, Université de Nantes, F-44000 Nantes, France \\ 4. CryoCapCell, Paris, 75015, France \\ 5. authors contributed equally to this work
}

Correlative Light and Electron Microscopy (CLEM) approaches allow linking molecular function to ultrastructure. One constant difficulty is the accurate correlation of structural identities of images with different magnifications and different contrast mechanisms in each modality.

Here, we present eC-CLEM (Easy Cell Correlative Light to Electron Microscopy) [1], provided as free open-source software implemented as a plugin in the Icy platform. Its purpose is to provide tools for 2D and 3D image correlation based on natural or artificial fiducial markers with local and global 3D correlation accuracy feedback. As correlation applies to a broad variety of data sets, eC-CLEM allows several type of registrations corresponding to different correlative workflows: from 3D LM stacks to 3D EM volumes or a mix of both - in order to reposition a slice in a stack or to extract a slice with the correct transformation from an LM stack. With eC-CLEM the registration can be done rigidly (Only scale, rotation and translation are applied) or non-rigidly (nonlinear transformations based on the spline transformation model), for 2D or 3D.

We also provide an estimation of the registration error in every point of the image, without ground truth based on statistical methods. From this error estimation, we provide an automatic feedback towards the best model for transformation to be used (rigid or non-rigid). In addition, eC-CLEM explores automatic registration through the 'AutoFinder' option: a step forward in CLEM automation [2]. 


\section{References:}

[1] Heiligenstein X et al, J. Nat Methods 14(2) (2017), p. 102.

[2] The authors thank the Icy team and, in particular, S. Dallongeville and F. de Chaumont, the Icy CODING party and France-BioImaging infrastructure supported by the French National Research Agency (ANR-10-INSB-04, "Investments for the future"). X.H. was funded by the Labex grant No. ANR-10-LBX-0038 part of the IDEX Idex PSL No. ANR-10-IDEX-0001-02 PSL. We acknowledge the FRM grant No. DEQ20140329491. MB is a member of the CryoCapCell company.
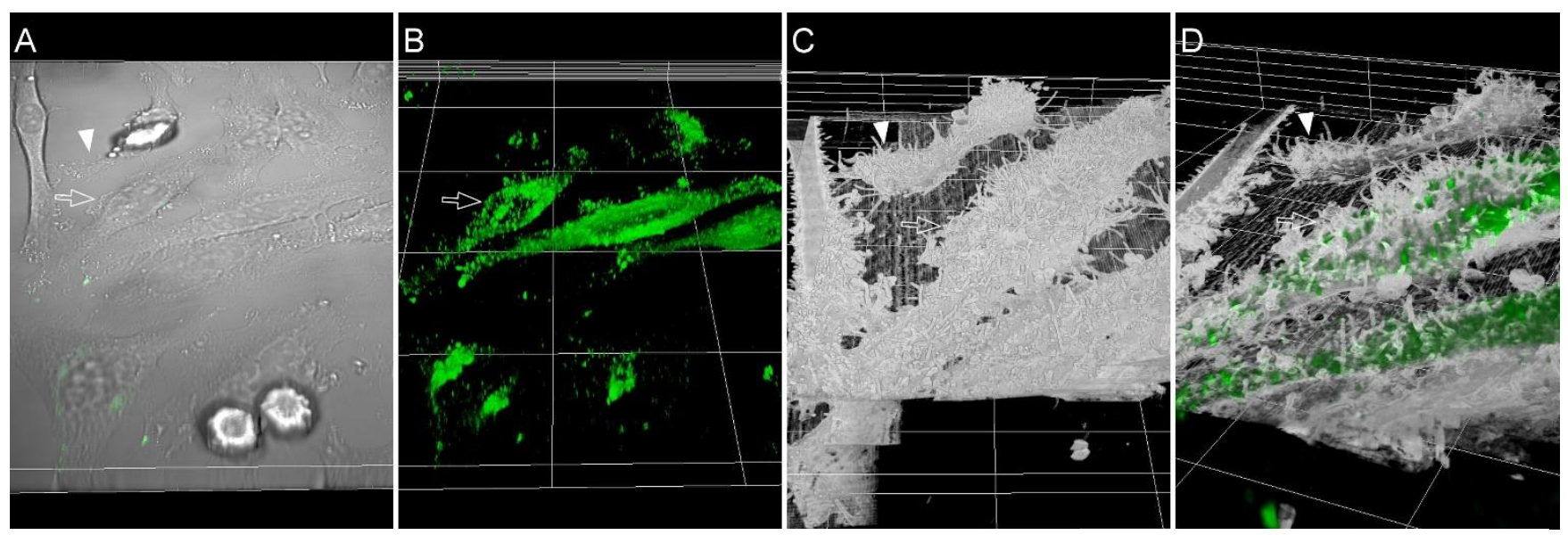

Figure 1. Example of 3D CLEM registration. A - Bright field light microscopy image (arrow head points towards non muted cell, outlined arrow points towards cell expressing a fluorescent protein). B - Same field by fluorescence light microscopy. C - Same field by Serial Bloc Face Scanning Electron Microscopy: cells expressing the fluorescent protein cannot be distinguished from the cells not expressing it. D - Image fusion of the light and electron microscopy pictures. 